S742.1099

March 19, 2018

\title{
A Note on Non-Commutative Orbifold Field Theories
}

\author{
Adi Armoni \\ Centre de Physique Théorique de l'École Polytechnique \\ 91128 Palaiseau Cedex, France \\ armoni@cpht.polytechnique.fr
}

\begin{abstract}
We suggest that orbifold field theories which are obtained from non-commutative $\mathcal{N}=4 \mathrm{SYM}$ are UV finite. In particular, nonsupersymmetric orbifold truncations might be finite even at finite values of $N_{c}$.
\end{abstract}




\section{Introduction}

Recently non-commutative Yang-Mills theory [1] attracted a lot of attention, mainly due to discoveries of new connections to string theory[2, 3, , 4]. In a recent paper [5], it was suggested that the divergences of the non-commutative Yang-Mills theory are dictated by the large $N_{c}$ limit of the theory. Namely, that divergences occur only in planar diagrams (the observation that the planar commutative and non-commutative theories are the same up to phases in the Green functions, was already made in [6]. A careful analysis of the divergences was carried out in ref.[7]).

Another direction of research is the study of orbifold field theories - motivated by the AdS/CFT correspondence [8]. It was conjectured by Kachru and Silverstein [9] that orbifolds of $A d S_{5} \times S^{5}$ which act on the $S^{5}$ part define a large $N_{c}$ finite theory, even when the R-symmetry is completely broken and the theory is not supersymmetric. This conjecture was later proved using both field theory 10 and string theory 11] techniques.

In this note we would like to consider non-commutative Yang-Mills theories which are obtained by an orbifold truncation of $\mathcal{N}=4 \mathrm{SYM}$. We suggest that these theories are UV finite, namely that there are no divergent Feynman diagrams, even when the theory under consideration is non-supersymmetric and the number of colors is finite. Note, however, that our conjecture relies on recent suggestions [5, 12] about UV finiteness of non-planar graphs in the non-commutative theory. The later were not fully proved yet, but seems to be necessary if the non-commutative theory is renormalizable.

\section{Orbifold field theories}

Orbifold field theories are obtained by a certain truncation of a supersymmetric Yang-Mills theory. Let us consider the special case of $\mathcal{N}=4$. The truncation procedure is as follows: consider a discrete subgroup $\Gamma$ of the $\mathcal{N}=4$ R-symmetry group $S U(4)$. For each element of the orbifold group, a representation $\gamma$ inside $S U\left(|\Gamma| N_{c}\right)$ should be specified. Each field $\Phi$ transform as $\Phi \rightarrow r \gamma^{\dagger} \Phi \gamma$, where $r$ is a representation matrix inside the R-symmetry group. The truncation is achieved by keeping invariant fields. The resulting theory has a reduced amount of supersymmetry, or no supersymmetry at all. It was conjectured [9], based on the AdS/CFT conjecture, that the truncated 
large $N_{c}$ theories are finite as the parent $\mathcal{N}=4$ theory. Later it was proved [10] that the planar diagrams of the truncated theory and parent theories are identical. In particular it means that the perturbative beta function of the large $N_{c}$ daughter theory is zero and that the theory is finite.

In the cases of $\mathcal{N}=2$ truncations, there is only one-loop (perturbative) contribution to the beta function. Its vanishing indicates the perturbative finiteness of the daughter theory at finite $N_{c}$ as well.

In $\mathcal{N}=1$ truncations the situation is more subtle. Indeed, the theory is finite at finite values of $N_{c}$, but the finiteness is due to Leigh-Strassler type of arguments [13]. In that case one should consider the $S U\left(N_{c}\right)$ version of the theory (and not the $U\left(N_{c}\right)$ theory which is obtained from the string theory orbifold), since the $U(1)$ beta function is always positive at the origin. In addition $\frac{1}{N_{c}^{2}}$ shifts of the Yukawa couplings are needed [14].

In the non-supersymmetric case there are no known examples of finite theories at finite $N_{c}$, though attempts in this direction using orbifolds were recently made[15]. As we shall see, due to non-commutativity such examples can be found.

\section{Perturbative behavior of non-commutative Yang-Mills theories}

Recently, several authors [7, 5] analyzed the renormalization behavior of noncommutative Yang-Mills theories (for an earlier discussion see[6]. Related works are [16, 17]). We briefly review their analysis. The Feynman rules of the commutative and non-commutative theories in momentum space are very similar. In fact the only difference is that each vertex of the noncommutative theory acquires a phase, $\exp i \Sigma_{i<j} k_{i} \wedge k_{j}$ (the Moyal phase), with respect to the vertex of ordinary commutative theory [6]. For planar diagrams, this phase cancels at internal loops and the only remnant is an overall phase. Therefore the planar commutative and non-commutative theories are similar, in accordance with recent findings [18, 19, 20]. In particular, thermodynamical quantities such as the free energy and the entropy are not affected by non-commutativity at the planar limit [19].

Another claim [5, 7] is that the oscillations of the Moyal phases at high mo-

mentum would regulate non-planar diagrams, namely that UV divergences 
of non-planar diagrams would disappear. There are two exceptional cases in which non-planar diagrams will still diverge[]: (i). When the non-planar diagrams consist of planar sub-diagrams which might diverge. (ii). For specific values of momentum (a zero measure set) the Moyal phase can be zero. Indeed, it was shown lately [12] that in theories which contain scalars - there are non-planar divergences. The theories that we will consider contain scalars and therefore contain infinities, however [12 interpret these divergences as Infra-Red divergences. The reason is as follows: The integrals which are associated with non-planar graphs converge unless the Moyal phase is set to zero by a vanishing incoming momentum. In this case new type of divergences appear, and it seems that infinite number of counterterms are needed. Therefore the non-commutative theory seems to be non-renormalizable. However, the authors of ref. 12] suggest that the re-summation of these divergent nonplanar graphs would yield a finite result. Their observation is based on the similarity between the present case and the standard (commutative) IR divergences. Note that Infra-Red divergences are, anyways, expected in theories with massless particles. Thus, 'truly' divergences in the non-commutative theory occur only in planar graphs.

\section{Finiteness of non-commutative orbifold field theories}

Let us now consider an orbifold truncation of non-commutative $\mathcal{N}=4 \mathrm{SYM}$. These theories can be defined perturbatively by a set of Feynman rules. The natural definition would be to attach to each vertex the corresponding Moyal phase [6]. According to ref. [5], the only potential divergences are the ones which arise in planar diagrams. Non-planar diagrams are expected to be finite, except for some "accidental" divergences [5]. Note that the infinities which do occur in these graphs are associated with the Infra-Red 12 and therefore do not contradict our claim that orbifold field theories are UV finite.

According to the analysis of [10], the planar diagrams of an orbifold theory can be evaluated by using the corresponding diagrams of the parent noncommutative $\mathcal{N}=4$. These diagrams are finite since they differ from the commutative $\mathcal{N}=4$ only by an overall phase. In this way sub-divergences of non-planar diagrams will also be canceled. We therefore conclude that any 
orbifold truncation of non-commutative non-compact $\mathcal{N}=4 \mathrm{SYM}$ is finite. In particular, it means that we might have a rich class of non-supersymmetric gauge theories which are finite, even at finite $N_{c}$ (in contrast to ordinary nonsupersymmetric orbifold field theories, where the two loop beta function is generically non-zero). Note that though these theories might be finite, they are certainly not conformal.

Let us consider a specific example 21]. The example is an $S U(N) \times S U(N)$ gauge theory with 6 scalars in the adjoint of each of the gauge groups and 4 Weyl fermions in the $(N, \bar{N})$ and 4 Weyl fermions in the $(\bar{N}, N)$ bifundamental representations. It is the theory which lives on dyonic D3 branes of type 0 string theory and can be also understood, from the field theory point of view, as a $Z_{2}$ orbifold projection of $\mathcal{N}=4 \mathrm{SYM} 22$. The large $N_{c}$ commutative theory contains a line of fixed points. We suggest that its analogous non-commutative (finite $N_{c}$ ) theory is finite at that line. Namely, at $g_{1}=g_{2}=h_{1}=h_{2}$, where $g_{1}, g_{2}$ are the gauge couplings and $h_{1}, h_{2}$ are the Yukawa couplings. Note that in contrast to the commutative case the position of the line of finite theories is not corrected by $\frac{1}{N_{c}}$ contributions.

Finally, it might be interesting to understand how the finiteness of these theories arise from string theory orbifolds.

\section{ACKNOWLEDGEMENTS}

I would like to thank O. Aharony, N. Itzhaki and B. Kol for useful discussions and comments. This work was supported in part by EEC TMR contract ERBFMRX-CT96-0090.

\section{References}

[1] A. Connes, Noncommutative Geometry. Academic Press, 1994.

[2] A. Connes, M. R. Douglas and A. Schwarz, "Noncommutative geometry and matrix theory: Compactification on tori", JHEP 02(1998) 003, hepth/9711162.

[3] M. R. Douglas and C. Hull, "D-branes and the noncommutative torus", JHEP 02 (1998) 008, hep-th/9711165. 
[4] N. Seiberg and E. Witten, "String Theory and Noncommutative Geometry", hep-th/9908142.

[5] D. Bigatti and L. Susskind, "Magnetic fields, branes and noncommutative geometry", hep-th/9908056.

[6] T. Filk, "Divergencies in field theory on quantum space", Phys.Lett. B376 (1996) 53.

[7] T. Krajewski and R. Wulkenhaar, "Perturbative Quantum Gauge Fields on The Noncommutative Torus", hep-th/9903187.

[8] J. M. Maldacena, "The Large $N$ Limit of Superconformal Field Theories and Supergravity", Adv.Theor.Math.Phys. 2 (1998) 231, hepth/9711200.

[9] S. Kachru and E. Silverstein, "4d Conformal Field Theories and Strings on Orbifolds", Phys.Rev.Lett. 80 (1998) 4855, hep-th/9802183.

[10] M. Bershadsky and A. Johansen, "Large $N$ limit of orbifold field theories", Nucl.Phys. B536 (1998) 141, hep-th/9803249.

[11] M. Bershadsky, Z. Kakushadze and C. Vafa, "String Expansion as Large $N$ Expansion of Gauge Theories", Nucl.Phys. B523 (1998) 59, hepth/9803076.

[12] S. Minwalla, M. Van Raamsdonk and N. Seiberg, "Noncommutative Perturbative Dynamics", hep-th/9912072.

[13] R. G. Leigh and M. J. Strassler, "Exactly Marginal Operators and Duality in Four Dimensional N=1 Supersymmetric Gauge Theory", Nucl.Phys. B447 (1995) 95, hep-th/9503121.

[14] Y. Oz and J. Terning, "Orbifolds of $A d S_{5} \times S^{5}$ and 4 d Conformal Field Theories", Nucl.Phys. B532 (1998) 163-180, hep-th/9803167.

[15] P. H. Frampton and W. F. Shively, "Conformal N=0 d=4 Gauge Theories from AdS/CFT Superstring Duality?", Phys.Lett. B454 (1999) 49, hep-th/9902168. 
[16] C. P. Martin and D. Sanchez-Ruiz, "The One-loop UV Divergent Structure of U(1) Yang-Mills Theory on Noncommutative $R^{4}$, Phys.Rev.Lett. 83 (1999) 476, hep-th/9903077.

[17] M. M. Sheikh-Jabbari, "One Loop Renormalizability of Supersymmetric Yang-Mills Theories on Noncommutative Two-Torus", JHEP 9906 (1999) 015 , hep-th/9903107.

[18] A. Hashimoto and N. Itzhaki, "Non-Commutative Yang-Mills and the AdS/CFT Correspondenc", hep-th/9907166.

[19] J. M. Maldacena and J. G. Russo, "Large N Limit of Non-Commutative Gauge Theories", hep-th/9908134.

[20] M. Li and Y.-S. Wu, "Holography and Noncommutative Yang-Mills", hep-th/9909085.

[21] I.R. Klebanov and A.A Tseytlin, "A Non-supersymmetric Large N CFT from Type 0 String Theory", JHEP 9903 (1999) 015, hep-th/9901101.

[22] N. Nekrasov and S. L. Shatashvili, "On Non-Supersymmetric CFT in Four Dimensions", hep-th/9902110. 